\title{
Intracranial Dural Arteriovenous Fistula: Preliminary Report of Arterial Spin Labeling
}

\author{
Kyo Noguchi*, Hideto Kawabe, Hikaru Seto \\ Department of Radiology, Toyama University, Toyama, Japan \\ Email: *kyo@med.u-toyama.ac.jp
}

Received December 26, 2012; revised January 30, 2013; accepted February 5, 2013

\begin{abstract}
Purpose: We report the results of applying arterial spin labeling (ASL) to intracranial dural arteriovenous fistula (DAVF). Methods: Sixteen patients with angiographically confirmed DAVF underwent ASL on a clinical $1.5 \mathrm{~T}$ or $3 \mathrm{~T}$ MR system. ASL was performed by Q2TIPS (second version of quantitative imaging of perfusion by using a single subtraction MRI pulse sequence with thin-slice TI1 periodic saturation) with echo-planar imaging. Draining veins such as dural venous sinus and cortical veins were evaluated on ASL. Results: ASL clearly depicted draining dural venous sinus in all patients with DAVF Borden type I (6/6, 100\%) and type II (4/4, 100\%). ASL depicted cortical venous reflux in only one patient with DAVF Borden type II (1/4, 25\%). ASL depicted drainage directly into cortical veins in all patients with Borden type III $(6 / 6,100 \%)$. Conclusion: Our preliminary study suggests that ASL can depict draining veins in patients with DAVF.
\end{abstract}

Keywords: Dural Arteriovenous Fistula; Arterial Spin Labeling; ASL

\section{Introduction}

Intracranial dural arteriovenous fistula (DAVF) is thought to be an acquired arteriovenous (AV) shunting disease, perhaps developing after venous sinus occlusion. DAVF is categorized as benign or aggressive depending on the pattern of venous drainage revealed by digital subtraction angiography (DSA) [1-5]. The DAVF classification schemes of Borden et al. and Cognard et al. are the most widely used $[3,4]$. Borden type I drains directly into the dural venous sinus, Borden type II drains into dural venous sinus with cortical venous reflux, while Borden type III drains directly into cortical veins. Borden type I is considered benign, whereas Borden type II and III are considered aggressive [3-5].

Arterial spin labeling (ASL) is an advanced MR perfusion imaging technique in which arterial blood water is electromagnetically labeled proximal to the brain and used as a diffusible flow tracer [6-9]. Recently, Le et al. [10] reported on venous ASL signal intensity seen frequently in 8 patients with DAVF and 7 patients with small AVM. We report the results of ASL applied to a larger group (16 patients) with DAVF including all Borden types.

\section{Materials and Methods}

\subsection{Patients}

Sixteen patients (12 men and 4 women; age range, 35 -

*Corresponding author.
78 years; mean age, 62 years) with angiographically confirmed DAVF were evaluated. The location of DAVF was at transverse-sigmoid sinus in 10 patients, tentorial sinus in 3 patients, superior sagittal sinus in 2 patients, and torcular herophili in one patient. The Borden classification of DAVF was type I in 6 patients, type II in 4 patients, and type III in 6 patients. Informed consent was obtained from all patients. MRI (ASL) and DSA were performed within 2 months of each other.

\subsection{MR Imaging and ASL}

MR imaging was performed on a clinical $1.5 \mathrm{~T}$ or $3 \mathrm{~T}$ MRI unit (Magnetom Verio or Avanto, Siemens AG, Erlangen, Germany) using a standard 12-channel head coil. 10 patients were examined at $1.5 \mathrm{~T}$ MRI, another 6 patients at 3 T MRI. ASL was performed according to a previously described technique [11]. The perfusion images were obtained by using Q2TIPS sequence (second version of quantitative imaging of perfusion by using a single subtraction MRI pulse sequence with thin-slice TI1 periodic saturation). Parameters of the ASL sequence were as follows: TR/TE/flip angel/slice number/acquisition time $=3000 \mathrm{~ms} / 16 \mathrm{~ms} / 90^{\circ} / 9 / 4 \mathrm{~min} 6 \mathrm{sec}$, and 2550 $\mathrm{ms} / 13 \mathrm{~ms} / 90^{\circ} / 9 / 4 \mathrm{~min} 6 \mathrm{sec}$, at $1.5 \mathrm{~T}$ and $3 \mathrm{~T}$ MRI, respectively. Inversion time 1 /saturation stop time/inversion time 2 was 700/1750/1800 ms. Field of view (FOV) was $220 \mathrm{~mm}$ and each voxel size was $3.4 \times 3.4 \times 8 \mathrm{~mm}^{3}$. Perfusion-weighted images (subtraction images) were 
used for evaluation in this study.

\subsection{Image Interpretation}

Two neuroradiologists, who had no background knowledge of patient clinical data or angiography findings independently reviewed the images. The identification of draining veins such as dural venous sinus or cortical veins on ASL was evaluated. Disagreements were resolved by consensus. These findings of marked hyperintense venous structures on ASL were defined as the signal intensity of venous structures clearly exceeding that of adjacent brain parenchyma.

\section{Results}

A summary of results showing identification of venous drainage on ASL in patients with DAVF is presented in Table 1. In all six patients with DAVF Borden type I, ASL depicted draining dural venous sinus as marked hyperintense areas. Marked hyperintense areas on ASL corresponded to draining dural venous sinus on DSA (Figures 1 and 2).

In all four patients with DAVF Borden type II, ASL depicted draining dural venous sinus as marked hyperintense areas. Marked hyperintense areas on ASL corresponded to draining dural venous sinus on DSA. However, ASL depicted cortical venous reflux as marked hyperintense areas in only one patient with DAVF Borden type II. In this patient with DAVF Borden type II, marked hyperintense areas on ASL corresponded to draining dural venous sinus and cortical venous reflux on DSA (Figure 3).

In all six patients with DAVF Borden type III, ASL depicted drainage directly into cortical veins as marked hyperintense areas, providing high lesion conspicuity. Marked hyperintense areas on ASL corresponded to drainage directly into cortical veins on DSA (Figures 4 and $5)$.

\section{Discussion}

This is a report on draining veins related to DAVF using ASL in a larger series of patients (16 patients) with DAVF including all Borden types. Our study suggests that ASL can depict draining veins such as dural venous sinus in patients with DAVF type I and II or cortical veins in patients with DAVF type III as marked hyperintensity.

Conventional MR imaging is typically normal in DAVF without retrograde cortical venous drainage and/or venous congestion. Conventional MR imaging is the procedure of choice for demonstrating the complications of DAVF, including venous infarction and hemorrhage [1214].

Three-dimensional time-of-flight (3D-TOF) MR angiography and MR digital subtraction angiography (MRDSA) are useful for non-invasive MR examinations for screening, before and after treatment, in DAVF [15-21]. Especially, 3D-TOF MRA is the most frequently used method of MRI examination for screening of DAVF because it does not require contrast media. However, 3D-TOF MRA is usually limited in the full evaluation of DAVF because of restrictions on FOV. The FOV of routine 3D-TOF MRA is usually placed to include transverse-sigmoid, cavernous sinuses, anterior condylar confluence, etc, but excludes most of the superior sagittal sinus. Therefore, routine 3D-TOF MRA is unsuited to screening for DAVF at superior sagittal sinus. ASL was able to depict DAVF at superior sagittal sinus in our study (Figure 3).

DAVF Borden type III is extremely aggressive [3-5]. The hemodynamics of DAVF-Borden type III is different from other types of DAVF. DAVF Borden type III does not drain into the sinus, as other types do, but directly into cortical veins, resembling that of arteriovenous malformation (AVM). AVM draining veins and nidus appear as marked hyperintensity structures representing rapid transit on ASL $[22,23]$. In our study, ASL clearly depicted drainage directly into cortical veins in DAVF Borden type III, providing high lesion conspicuity (Figures 4 and 5).

ASL uses labeled arterial blood as diffusible water that is $90 \%$ extracted on the first pass through the capillary bed. Therefore, by using ASL (Q2TIPS) in which arteries are labeled, the ASL marked hyperintense area is not typically seen in the venous system except in the presence of arteriovenous connections such as AVM or DAVF. In our study, it was difficult for ASL (Q2TIPS) to depict

Table 1. Identification of venous drainage of DAVF on ASL.

\begin{tabular}{cccc}
\hline \multirow{2}{*}{ Classification (Borden) } & Number of case & Identification of venous drainage on ASL \\
\cline { 3 - 4 } & & Dural venous sinus & Cortical veins \\
\hline Type I & 6 & $6 / 6(100 \%)$ & N/A \\
Type II & 4 & $4 / 4(100 \%)$ & $6 / 6(100 \%)$ \\
Type III & 6 & N/A & 6 \\
\hline
\end{tabular}

Note: N/A indicates not applicable. 

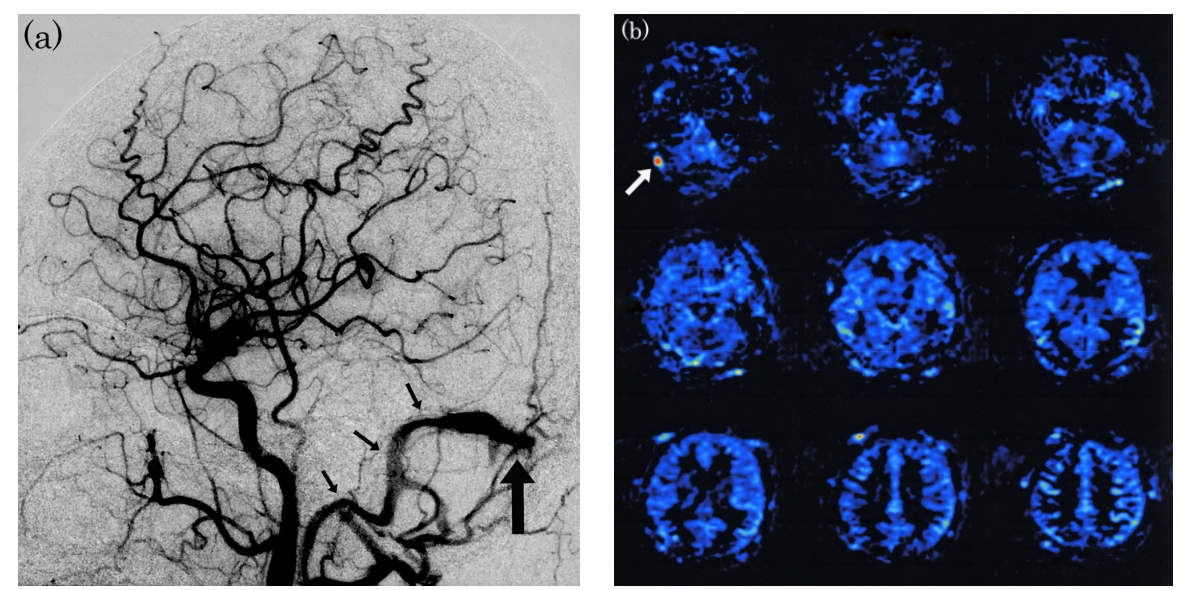

Figure 1. Dural arteriovenous fistula (Borden type I) in a 61-year-old man. (a) Arterial phase of right common carotid angiogram showed dural arteriovenous fistula at right transverse sinus (large arrow) with drainage into right transverse-sigmoid sinus (small arrows); (b) ASL shows hyperintense area (arrow) in right transverse-sigmoid sinus, indicating the presence of arteriovenous shunting.
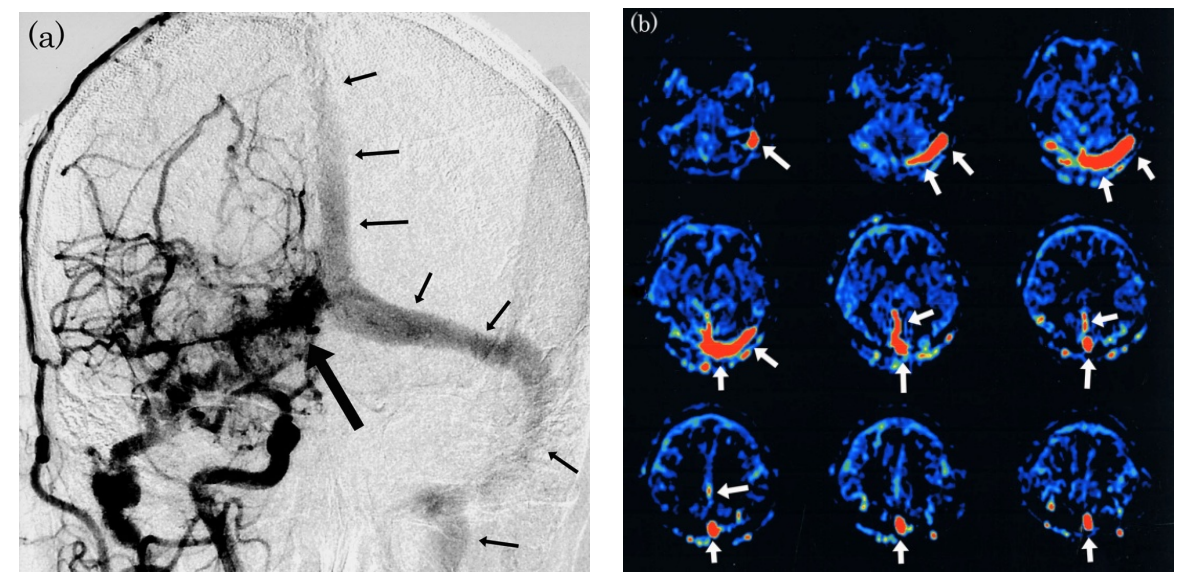

Figure 2. Dural arteriovenous fistula (Borden type I) in a 72-year-old man. (a) Arterial phase of right common carotid angiogram shows dural arteriovenous fistula at right transverse sinus (large arrow) with right transverse-sigmoid sinus occlusion and reflux into left transverse-sigmoid and superior sagittal sinuses (small arrows); (b) ASL shows marked hyperintense areas (arrows) in left transverse-sigmoid, superior sagittal and straight sinuses, indicating the presence of arteriovenous shunting.
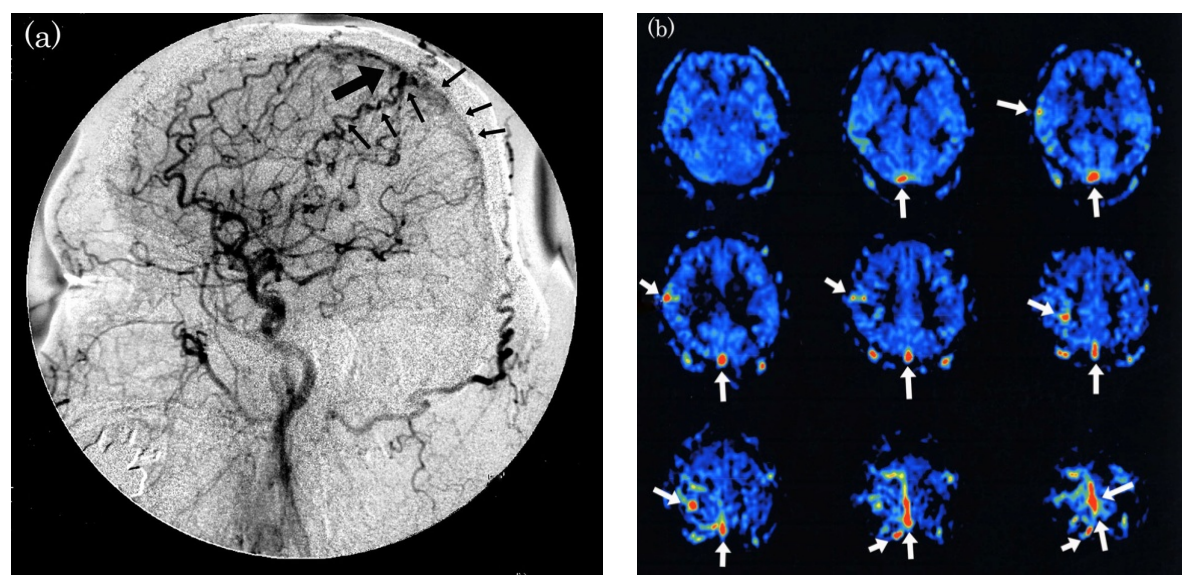

Figure 3. Dural arteriovenous fistula (Borden type II) in a 48-year-old man. (a) Arterial phase of right common carotid angiogram shows dural arteriovenous fistula at superior sagittal sinus (large arrow) with drainage into superior sagittal sinus (small arrows) and cortical venous reflux (small arrows); (b) ASL shows hyperintense areas (arrows) in superior sagittal sinus and right cortical vein, indicating the presence of arteriovenous shunting. 

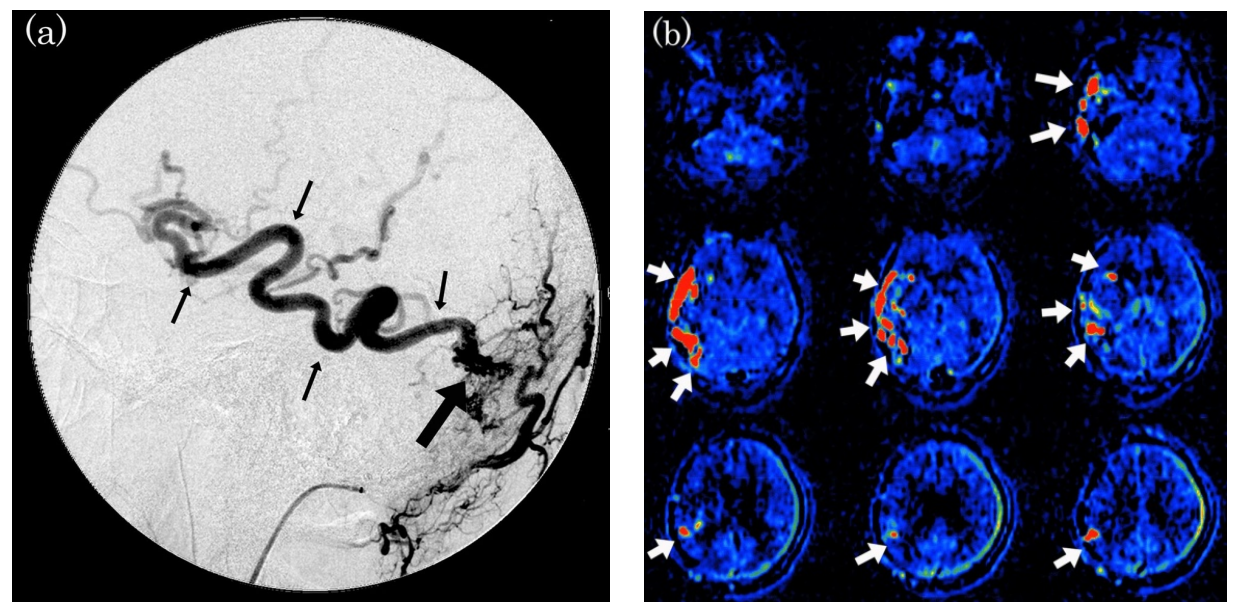

Figure 4. Dural arteriovenous fistula (Borden type III) in a 56-year-old man. (a) Arterial phase of right external carotid angiogram shows dural arteriovenous fistula at right transverse-sigmoid sinus (large arrow) with drainage directly into right vein of Labbe (small arrows); (b) ASL shows marked hyperintense areas (arrows) in the right cortical vein, indicating the presence of arteriovenous shunting.
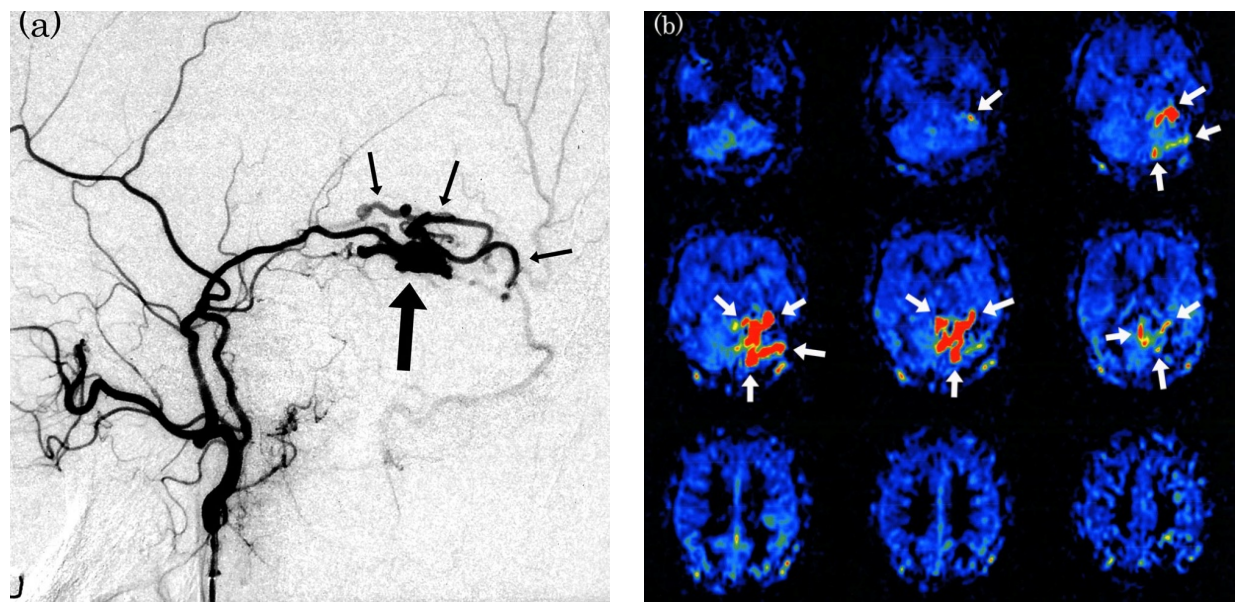

Figure 5. Dural arteriovenous fistula (Borden type III) in a 65-year-old man. (a) Arterial phase of left external carotid angiogram shows dural arteriovenous fistula at left tentorial sinus (large arrow) with drainage directly into cortical veins (small arrows); (b) ASL shows marked hyperintense areas (arrows) around left tentorium cerebelli, indicating the presence of arteriovenous shunting.

cortical venous reflux in DAVF Borden type II. The magnitude of the venous ASL signal intensity depends on the number of spins transversing the fistula, and both labeling time and delay time. On the other hand, other ASL such as FAIR (with single slice) can depict cortical venous reflux because both arteries and veins are labeled [24].

In this study, it was difficult for ASL (Q2TIPS) to depict cortical venous reflux in DAVF Borden type II. Our study suggested that it is difficult to clearly differentiate Borden type I from type II on ASL alone. Therefore, the combination of other MR imaging methods may help to differentiate this condition. Recent reports have suggested that some advanced MR imaging techniques such as dynamic susceptibility contrast (DSC) and susceptibility-weighted imaging (SWI) are capable of detecting and evaluating cortical venous reflux and venous congestion in patients with DAVF [25-27]. Further studies may be necessary to assess the value of the combination of ASL and other advanced MR imaging such as DSC and SWI for the demonstration of cortical venous reflux in DAVF.

However, MR imaging including 3D-TOF MRA, MRDSA and advanced MR imaging cannot replace DSA because it provides insufficient hemodynamics and spatial resolution. DSA is still the gold standard for precise evaluation of DAVF before and after treatment.

Our study has some important limitations. First, it was difficult for ASL to depict cortical venous reflux in DAVF Borden type II, as previously described. Second, the site of DAVF was limited. Most cases in this study were DAVF at the transverse-sigmoid sinus. Third, it may be difficult for ASL to depict DAVF with a very 
small AV-shunt. Further studies with larger subject groups, including DAVF at any site with varying degrees of AVshunting and larger treated cases, will be required to fully assess this technique.

\section{Conclusion}

Our study suggested that ASL could depict draining veins in patients with DAVF. Especially, ASL clearly depicted drainage directly into cortical veins in DAVF Borden type III, providing high lesion conspicuity. ASL may provide useful non-invasive supplementary MR imaging for rough assessment in patients with DAVF.

\section{REFERENCES}

[1] P. Lasjaunias, M. Chiu, K. TerBrugge, A. Tolia, M. Hurth and M. Bernstein, "Neurological Manifestations of Intracranial Dural Arteriovenous Malformations,” Journal of Neurosurgery, Vol. 64, No. 5, 1986, pp. 724-730. doi:10.3171/jns.1986.64.5.0724

[2] I. Awad, J. Little, W. Akrawi and J. Ahl, "Intracranial Dural Arteriovenous Malformations: Factors Predisposing to an Aggressive Neurological Course,” Journal of Neurosurgery, Vol. 72, No. 6, 1990, pp. 839-850. doi:10.3171/jns.1990.72.6.0839

[3] J. A. Borden, J. K. Wu and W. A. Shucart, “A Proposed Classification for Spinal and Cranial Dural Arteriovenous Fistulous Malformations and Implications for Treatment," Journal of Neurosurgery, Vol. 82, No. 2, 1995, pp. 166179. doi:10.3171/jns.1995.82.2.0166

[4] C. Cognard, Y. Gobin, L. Pierot, et al., "Cerebral Dural Arteriovenous Fistulas: Clinical and Angiographic Correlation with a Revised Classification of Venous Drainage,” Radiology, Vol. 194, 1995, pp. 671-680.

[5] J. M. van Dijk, K. G. terBrugge, R. A. Willinsky and M. C. Wallace, "Clinical Course of Cranial Dural Arteriovenous Fistulas with Long-Term Persistent Cortical Venous Reflux,” Stroke, Vol. 33, No. 5, 2002, pp. 12331236. doi:10.1161/01.STR.0000014772.02908.44

[6] A. R. Deibler, J. M. Pollock, R. A. Kraft, H. Tan, J. H. Burdette and J. A. Maldjian, "Arterial Spin-Labeling in Routine Clinical Practice, Part 1: Technique and Artifacts," American Journal of Neuroradiology, Vol. 29, No. 7, 2008, pp. 1228-1234. doi:10.3174/ajnr.A1030

[7] A. R. Deibler, J. M. Pollock, R. A. Kraft, H. Tan, J. H. Burdette and J. A. Maldjian, “Arterial Spin-Labeling in Routine Clinical Practice, Part 2: Hypoperfusion Patterns," American Journal of Neuroradiology, Vol. 29, 2008, 235-1241. doi:10.3174/ajnr.A1033

[8] A. R. Deibler, J. M. Pollock, R. A. Kraft, H. Tan, J. H. Burdette and J. A. Maldjian, "Arterial Spin-Labeling in Routine Clinical Practice, Part 3: Hyperperfusion Patterns," American Journal of Neuroradiology, Vol. 29, 2008, pp. 1428-1435. doi:10.3174/ajnr.A1034

[9] E. T. Petersen, I. Zimine, Y. C. Ho and X. Golay, "NonInvasive Measurement of Perfusion: A Critical Review of Arterial Spin Labeling Techniques,” The British Journal of Radiology, Vol. 79, No. 944, 2006, pp. 688-701. doi:10.1259/bjr/67705974

[10] T. T. Le, N. J. Fischbein, J. B. Andre, C. Wijman, J. Rosenberg and G. Zaharchuk, "Identification of Venous Signal on Arterial Spin Labeling Improves Diagnosis of Dural Arterioveninous Fistulas and Small Arteriovenous Malformations,” American Journal of Neuroradiology, Vol. 33, 2012, pp. 61-68. doi:10.3174/ajnr.A2761

[11] W. M. Luh, E. C. Wong, P. A. Badettini and J. S. Hyde, "QUIPSS II with Thin-Slice TI1 Periodic Saturation: A Method for Improving Accuracy of Quantitative Perfusion Imaging Using Pulsed Arterial Spin Labeling,” Magnetic Resonance in Medicine, Vol. 41, No. 6, 1996, pp. 1246-1254. doi:10.1002/(SICI)1522-2594(199906)41:6<1246::AIDMRM22>3.0.CO;2-N

[12] K. DeMarco, W. P. Dillon, V. V. Halbach and J. S. Tsuruda, "Dural Arteriovenous Fistula: Evaluation with MR Imaging,” Radiology, Vol. 175, No. 1, 1990, pp. 193-199.

[13] R. Willinsky, K. Terbrugge, W. Montanera, D. Mikulis and C. Wallace, "Venous Congestion: An MR Finding in Dural Arteriovenous Malformations with Cortical Venous Drainage,” American Journal of Neuroradiology, Vol. 15, 1994, pp. 1501-1507.

[14] R. Willinsky, M. Goyal, K. terBrugge and W. Montanera, "Tortuous, Enlarged Pial Veins in Intracranial Dural Arteriovenous Fistulas: Correlations with Presentation, Location, and MR Findings in 122 Patients,” American Journal of Neuroradiology, Vol. 20, No. 6, 1996, pp. 10311036.

[15] J. C. Chen, J. S. Tsuruda and V. V. Halbach, "Suspected Dural Arteriovenous Fistula: Results with Screening MR Angiography in Seven Patients," Radiology, Vol. 183, 1992, pp. 265-271.

[16] K. Noguchi, E. R. Melhem, T. Kanazawa, M. Kubo, N. Kuwayama and H. Seto, "Intracranial Dural Arteriovenous Fistulas: Evaluation with Combined 3D Time-ofFlight MR Angiography and MR Digital Subtraction Angiography," American Journal of Roentgenology, Vol. 182, No. 1, 2004, pp. 183-190.

[17] S. C. Coley, C. A. J. Romanowski, T. J. Hodgson and P. D. Griffiths, "Dural Arteriovenous Fistulae: Noninvasive Diagnosis with Dynamic MR Digital Subtraction Angiography," American Journal of Neuroradiology, Vol. 23, 2002, pp. 404-407.

[18] N. Horie, M. Morikawa, N. Kitigawa, K. Tsutsumi, M. Kaminogo and I. Nagata, “2D Thick-Section MR Digital Subtraction Angiography for the Assessment of Dural Arteriovenous Fistulas," American Journal of Neuroradiology, Vol. 27, No. 2, 2006, pp. 264-269.

[19] K. Noguchi, N. Kuwayama, M. Kubo, et al., "Dural Arteriovenous Fistula Involving the Transverse Sigmoid Sinus after Treatment: Assessment with Magnetic Resonance Digital Subtraction Angiography,” Neuroradiology, Vol. 49, No. 8, 2007, pp. 639-643. doi:10.1007/s00234-007-0230-4

[20] R. I. Farb, R. Agid, R. A. Willinsky, D. M. Johnstone and K. G. terBrugge, "Cranial Dural Arteriovenous Fistula: Diagnosis and Classification with Time-Resolved MR 
Angiography at 3 T," American Journal of Neuroradiology, Vol. 30, No. 8, 2009, pp. 1546-1551.

doi:10.3174/ajnr.A1646

[21] S. Nishimura, T. Hirai, A. Sasao, et al., "Evaluation of Dural Arteriovenous Fistulas with 4D Contrast-Enhanced MR Angiography at 3 T,” American Journal of Neuroradiology, Vol. 31, No. 1, 2010, pp. 80-85. doi:10.3174/ajnr.A1898

[22] R. L. Wolf, J. Wang, J. A. Detre, E. L. Zager and R. W. Hurst, "Arteriovenous Shunt Visualization in Arteriovenous Malformations with Arterial Spin-Labeling MR Imaging," American Journal of Neuroradiology, Vol. 29, 2008, pp. 681-687. doi:10.3174/ajnr.A0901

[23] J. M. Pollock, C. T. Whitlow, J. Simonds, et al., "Response of Arteriovenous Malformations to Gamma Knife Therapy Evaluated with Pulsed Arterial Spin-Labeling MRI Perfusion," American Journal of Roentgenology, Vol. 196, No. 1, 2011, pp. 15-22. doi:10.2214/AJR.10.5290

[24] K. Noguchi, N. Kuwayama, M. Kubo, et al., "Flow-Sensitive Alternating Inversion Recovery (FAIR) Imaging for Retrograde Cortical Venous Drainage Related to Intrac- ranial Dural Arteriovenous Fistula,” Neuroradiology, Vol. 53, No. 3, 2011, pp. 153-158.

doi:10.1007/s00234-010-0711-8

[25] K. Noguchi, N. Kuwayama, M. Kubo, et al., "Intracranial Dural Arteriovenous Fistula with Retrograde Cortical Venous Drainage: Use of Susceptibility-Weighted Imaging in Combination with Dynamic Susceptibility Contrast Imaging," American Journal of Neuroradiology, Vol. 31, 2010, pp. 1903-1910. doi:10.3174/ajnr.A2231

[26] J. Hodel, S. Gerber, M. Zins, M. Rodallec, X. Leclerc, R. Blanc and A. Rahmouni, "MR Imaging Findings in Intracranial Dural Arteriovenous Shunt with Retrograde Cortical Venous Drainage Using Susceptibility-Weighted Angiography," American Journal of Neuroradiology, Vol. 32, 2011, pp. 196-197. doi:10.3174/ajnr.A2875

[27] L. Letourneau-Guillon and T. Krings, "Simultaneous Arteriovenous Shunting and Venous Congestion Identification in Dural Arteriovenous Fistulas Using SusceptibilityWeighted Imaging: Initial Experience,” American Journal of Neuroradiology, Vol. 33, 2012, pp. 301-307. doi:10.3174/ajnr.A2777 